TITLE:

\title{
Thenar Dysplasia in Radial Polydactyly Depends on the Level of Bifurcation
}

\section{$\operatorname{AUTHOR}(\mathrm{S}):$}

Saito, Susumu; Ueda, Maho; Murata, Mai; Suzuki, Shigehiko

\section{CITATION:}

Saito, Susumu ... [et al]. Thenar Dysplasia in Radial Polydactyly Depends on the Level of Bifurcation. Plastic and Reconstructive Surgery 2018, 141(1): 85e-90e

\section{ISSUE DATE:}

2018-01-01

URL:

http://hdl.handle.net/2433/228917

\section{RIGHT:}

This is a non-final version of an article published in final form in (provide complete journal citation); The full-text file will be made open to the public on 01 January 2019 in accordance with publisher's 'Terms and Conditions for SelfArchiving'.; この論文は出版社版でありません。引用の際には出版社版をご確認ご利用ください。; This is not the published version. Please cite only the published version. 
“Thenar Dysplasia in Radial Polydactyly Depends on the Level of Bifurcation."

Author list: Susumu Saito, MD, PhD ${ }^{1}$; Maho Ueda, MD ${ }^{1}$; Mai Murata, MD ${ }^{1}$; Shigehiko Suzuki, MD, $\mathrm{PhD}^{1}$.

1. Department of Plastic and Reconstructive Surgery, Graduate School of Medicine and Faculty of Medicine, Kyoto University. 


\section{Corresponding author:}

Susumu Saito MD, PhD.

Yoshida-Konoe-cho, Sakyo-ku

Kyoto, Japan

Postal code: 606-8501

Telephone number: + 81-75-751-3613

Fax number: +81-75-751-4340

e-mail address: $\underline{\text { susumus@kuhp.kyoto-u.ac.jp }}$ 
Financial Disclosure Statement: No funding was received for this article. 
This study is registered in UMIN Clinical Trials Registry

Trial registry name：“カスタムメイド超音波 $3 \mathrm{D}$ 画像による体表解剖に関する研究および 臨床診断における有効性に関する研究” (Studies on superficial anatomies of human body by $3 \mathrm{D}$ ultrasound)

Registration identification number: UMIN000014396

URL: https://upload.umin.ac.jp/cgi-open-bin/ctr/ctr_view.cgi?recptno=R000016753 
This study was accepted to be orally presented at $4^{\text {th }}$ European Symposium on Pediatric Hand Surgery and Rehabilitation, which will be held in Paris, France in June 15-16, 2017.

Short Running Head

Thumb Dysplasia in Radial Polydactyly 
Abstract

\section{Background}

Little is known about thenar dysplasia in radial polydactyly, other than that thenar hypoplasia occasionally associated with thenar dysplasia remains unclear.

\section{Methods}

10 The abductor pollicis brevis (APB) and flexor pollicis brevis (FPB) muscles were visualized using

11 three-dimensional ultrasound and their horizontal geometry was assessed using a biaxial level classification system. Subjects were categorized into three phenotypes according to the developmental

13 condition of the radial thumb. The relationship between the level of distribution of the muscles and the level

14 of the bifurcation of the radial thumb was investigated.

\section{$15 \quad$ Results}

16 Nineteen patients with radial polydactyly without triphalangism were included. There were ten patients with

17 the non-floating type, three with the floating type, and six with the rudimentary type. All patients with

18 bifurcation at or more distal to the metacarpophalangeal (MCP) joint had normal thenar muscle distribution, 
19 but the muscles in patients with bifurcation at or more proximal to the level of the metacarpals were

20 confined, regardless of phenotype. The level of muscle distribution was strongly correlated with the level of

21 the bifurcation of the radial thumb.

\section{Conclusions}

23 These findings suggest that formation of the thenar muscles in the longitudinal direction in radial polydactyly

24 might depend on the level of bifurcation of the radial thumb. The presence of thenar dysplasia even in floating-type or rudimentary-type duplications is of clinical and etiological importance.

Key Words

Radial polydactyly, thenar muscle, dysplasia, ultrasound 
31 For radial polydactyly, reconstructive surgery has been widely accepted as a result of increased knowledge about associated skeletal and soft tissue abnormalities ${ }^{1-4}$. However, even though all these anomalies are addressed intraoperatively, complete functional restoration of the thumb remains challenging, ${ }^{5,}$. In 1982 ,

Miura stated issues remaining after reconstruction for radial polydactyly are adduction contracture of the thumb and thenar dysplasia ${ }^{7}$. Although more than 30 years have passed since Miura's statement, little is known about thenar dysplasia in radial polydactyly apart from the fact that thenar hypoplasia occurs in special cases of radial polydactyly with triphalangism ${ }^{8}$. polydactyly without triphalangism using three-dimensional (3D) ultrasonography. In particular, the relationship between thenar dysplasia and the level of bifurcation of the radial thumb was evaluated.

\section{Materials and methods}

This study began in 2014 after it was approved by the ethics committee of our institution. The patients' families received an explanation about the 3D ultrasound examination and surgery, and written informed consent was obtained. Triphalangeal-thumb polydactyly or polysyndactyly, and a six-fingered hand were not included because these types were known to have distinct pathogenic backgrounds ${ }^{9,10}$. 
under precise speed regulation at $3 \mathrm{~mm} / \mathrm{s}$. During scan, the thumb was placed in the 45 -degree

radially-abducted position. Consecutive images were acquired at $0.2 \mathrm{~mm}$ intervals and processed to

reconstruct 3D data using imaging software (ImageJ, U. S. National Institutes of Health (NIH), Bethesda,

Maryland, USA). Anatomical identifications were accomplished on the basis of multi-planar observation,

initially using coronal sections, followed by sagittal and axial sections. Especially for coronal sections, the

outlines of the first metacarpal were memorized to be shown in more superficial sections as anatomical

references. (Supplementary figure. 1).

To systematically investigate developmental conditions of the thenar muscles in patients with radial

polydactyly of various degrees of severity and phenotypes, subjects were categorized according to the level

of bifurcation and the developmental condition of the radial thumb. For bifurcation, nine levels were defined

along the phalanges and metacarpal (Fig. 1). For developmental condition of the radial thumb, three

phenotypes were defined. The non-floating type had a true articulation or an osseous continuity between 
respectively. The APB distribution was assessed only on the insertion side by levels along the metacarpal

axis. The relationship between the level of muscle distribution and the level of the bifurcation of the radial thumb was analyzed using Pearson's correlation coefficients for samples bifurcating at Levels 1-7 because the levels from Level 1 to Level 7 were aligned regularly.

\section{Results}

Consecutive nineteen patients were included in this study. There were no bilateral cases. Additional examinations using nine unaffected hands of infants verified that the APB on the insertion side, FPB on the insertion side, and FPB on the origin side located at Levels 6,6 , and 5, respectively. Scatter plots of the distribution of the thenar muscles are shown in Fig. 2. In all hands with Level 6 or more distal bifurcation, muscle distribution was within the normal range for both APB and FPB regardless of phenotypic differences in the radial thumb (Supplementary figure 2). In contrast, in hands with Level 5 or more proximal bifurcation, the level of muscle distribution was strongly related to the level of the bifurcation, especially for APB and FPB on the insertion side. Notably, even the origin side of the FPB was distributed proximally according to the proximity of the bifurcation of the radial thumb, especially in cases with Level 3 or more proximal bifurcation. Representative cases were presented in Supplementary figure 3-6.

\section{Discussion}


84 longitudinal direction is limited by the level of bifurcation. Surprisingly, proximal confinement of the thenar

muscles was found even in patients with a metacarpal duplication of the floating or rudimentary type,

suggesting that the mechanism we proposed may apply to different phenotypes of radial polydactyly,

although the presence of pathogenic factors in the transverse direction is speculated (Fig. 3). These results

provide two-fold clinical implications. One is that aberrant APB and FPB insertion can exist even in floating

and rudimentary types. The other is that longitudinal underdevelopment of the thenar muscles can influence

surgical outcomes. Lourie et al. reported unusual cases showing a zigzag deformity after ligation treatment

for an extra pedunculated thumb with at the radial aspect of the metacarpal ${ }^{12}$. Similarly, we experienced 
104 All the authors contributed to the writing of the paper. 
107 References

108 1. Marks TW and Bayne LG. Polydactyly of the thumb: Abnormal anatomy and treatment. J Hand Surg.

$109 \quad 1978 ; 3: 107-116$.

110 2. Tada K, Yonenobu K, Tsuyuguchi Y, Kawai H, Egawa T. Duplication of the thumb. A retrospective review

111 of two hundred and thirty-seven cases. J Bone Joint Surg Am. 1983; 65:584-98.

112 3. Miura T. An appropriate treatment for postoperative Z-formed deformity of the duplicated thumb. J Hand

113 Surg. 1977; 2:380-386.

114 4. Salama R, Weissman SL. Congenital bilateral anomalous band between flexor and extensor pollicis

115 longus tendons. Report of a case. Hand. 1975; 7:25-26.

116 5. Kawabata H, Tada K, Masada K, Kawai H, Ono K. Revision of residual deformities after operations for

117 duplication of the thumb. J Bone Joint Surg Am. 1990; 72:988-998.

118 6. Naasan A, Page RE. Duplication of the thumb. A 20-year retrospective review. J Hand Surg Br. 1994;

$119 \quad 19: 355-360$.

120 7. Miura T. Duplicated thumb. Plast Reconstr Surg. 1982; 69:470-481.

121 8. Graham JM, Jr., Brown FE and Hall BD. Thumb Polydactyly as a Part of the Range of Genetic

122 Expression for Thenar Hypoplasia. Clin Pediatr (Phila). 1987; 26:142-148.

123 9. Wieczorek D, Pawlik B, Li Y, Akarsu NA, Caliebe A, May KJ, et al. A specific mutation in the distant sonic 

hedgehog (SHH) cis-regulator (ZRS) causes Werner mesomelic syndrome (WMS) while complete ZRS thumb. Hum Mutat. 2010; 31:81-89. enhancer. J Med Genet. 2008; 45:589-595. Visualization of Muscular Anomalies in Type VI and VII Radial Polydactyly. Skel Radiol. 2016; 45:541-547. 12. Lourie GM, Costas BL, Bayne LG. The zig-zag deformity in pre-axial polydactyly. A new cause and its treatment. J Hand Surg Br. 1995; 20:561-564. of Upper Limb Modified by the Japanese Society for Surgery of the Hand (JSSH). Hand Surg. 2015; 
142 study of two classification systems for radial polydactyly. Plast Reconstr Surg. 2014; 134:991-1001. 
$144 \quad$ Figure legends

145

$146 \quad$ Fig. 1

147 The biaxial level classification system and its application

148 Seven levels are defined for the thenar region along two anatomical axes. In addition to the seven levels,

149 two levels are defined distally in order to classify the level of bifurcation of the radial thumb (top). An

150 ultrasound coronal image of a non-floating-type duplication with Level 5 bifurcation (bottom). In this case,

151 the APB on the insertion side, FPB on the insertion side, and FPB on the origin side all distribute at Levels 5.

152 APB, abductor pollicis brevis; FPB, flexor pollicis brevis; CMC, carpometacarpal; MCP,

153 metacarpophalangeal; IP, interphalangeal.

Fig. 2

156 Scatter plots and lines of best fit showing relationships between distributions of APB and FPB versus the 
162 Schematic illustration showing a proposed mechanism of thenar dysplasia associated with radial

163 polydactyly.

164

165

Supplementary figure 1

166

Ultrasound images showing normal thenar anatomy in a 1-year-old infant. A, coronal; B, sagittal; C, axial

sections. Lines with numbers indicate the location of the images. Green lines indicate the outline of the first

metacarpal. Yellow lines show the outline of the thenar muscles. FPL is used as a landmark that separates

the superficial and deep part of the muscles (Red circles). APB, abductor pollicis brevis; AD, adductor

pollicis; FPB, flexor pollicis brevis; FPL, flexor pollicis longus; OP, opponens pollicis; CT, carpal tunnel; CMC,

carpometacarpal joint; MCP, metacarpophalangeal joint; $1^{\text {st }} \mathrm{MC}$, first metacarpal; $d$, depth from the skin.

Supplementary figure 2

or more distally. Yellow lines show the outline of the superficial thenar muscles. Note that all the muscles indicated in Fig. 2. 
180 A 6-month-old patient with a non-floating-type duplication with Level 1 bifurcation. A, The thenar eminence

181 seems hypoplastic (right). Note that the proximal thenar crease is lacking (arrow). B, Radiograph. C,

182 Coronal; D, axial; and E, sagittal ultrasound sections. The thenar muscles are confined proximally to Level

1832 (red arrowheads). The distal portion of the muscles is located in the direction of the MCP joint of the radial

184 thumb (yellow arrowheads). Yellow lines show the outline of the thenar muscles. Red circles indicate the

185 FPL in a cross-sectional view. Abbreviations are as indicated in Fig. 2. L and R indicate left and right,

186 respectively.

187

188

Supplementary figure 4

189 A 1-year-old patient with a floating-type extra thumb bifurcating at Level 3. A, The thenar eminence seems

190 slightly hypoplastic (right, arrow). B, Radiograph. C, Coronal; D, axial; and E, sagittal ultrasound sections.

191 The thenar muscles are confined proximally to Level 3 (red arrowheads). Note that there is no muscle

192 distribution in the direction of the MCP joint of the ulnar thumb (yellow arrowheads). Yellow lines show the

193 outline the thenar muscles. Red circles indicate the FPL in a cross-sectional view. Abbreviations used are

194 indicated in Fig. 2. L and R indicate left and right, respectively.

195

196

Supplementary figure 5

197 A 4-year-old patient who underwent ablative surgery for rudimentary-type duplication with Level 4 
198 bifurcation four years previously. A, The thenar eminence seems underdeveloped radially (arrows). Palmar

199 abduction of the affected thumb is insufficient (bottom, right). B, Preoperative appearance (left) and

200 radiograph (right). C, Coronal; D, sagittal; and E, axial ultrasound sections. Axial sections at the level of the

201 epiphyseal line demonstrate APB atrophy (red arrowheads) although FPB is shown is less underdeveloped

202 (yellow arrowheads). Red circles indicate the FPL in a cross-sectional view. Abbreviations used are

203 indicated in Fig. 2. L and R indicate left and right, respectively.

Supplementary figure 6

206 A 14-year-old patient with untreated rudimentary-type duplication with Level 2 bifurcation. A, The thenar eminence seems underdeveloped (top, black arrow). The involved thumb shows a deformity in the flexion position (bottom, white arrow). B, Radiograph. C, Coronal; D, sagittal; and E, axial ultrasound sections. The thenar muscles are confined proximally to Level 2 (red arrowheads). Axial sections at the level of the epiphysis demonstrate muscle atrophy (yellow arrowheads). Yellow lines show the outline of the thenar muscles. Red circles indicate the FPL in a cross-sectional view. Abbreviations used are indicated in Fig. 2. L 
Fig. 1
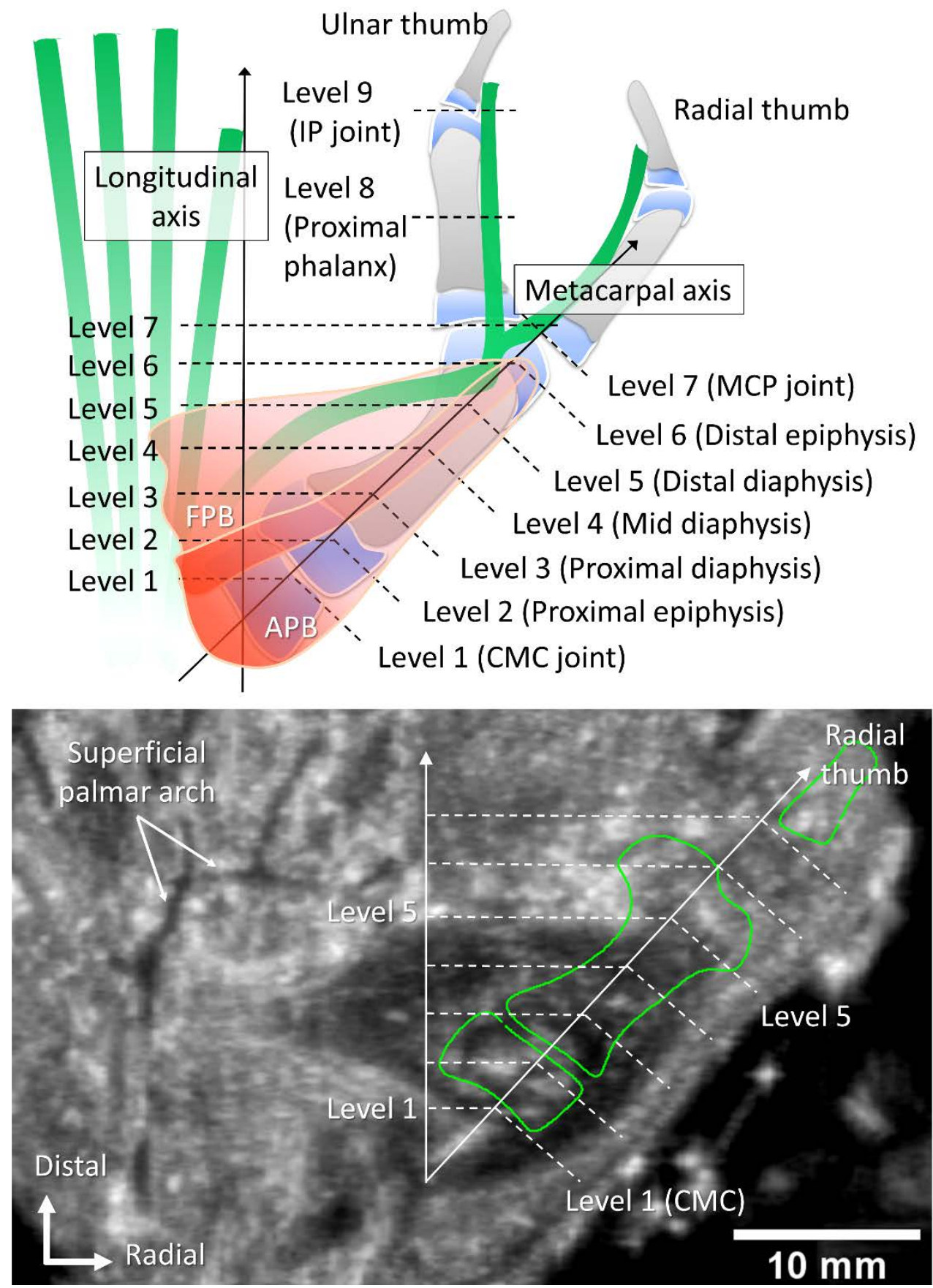
Fig. 2
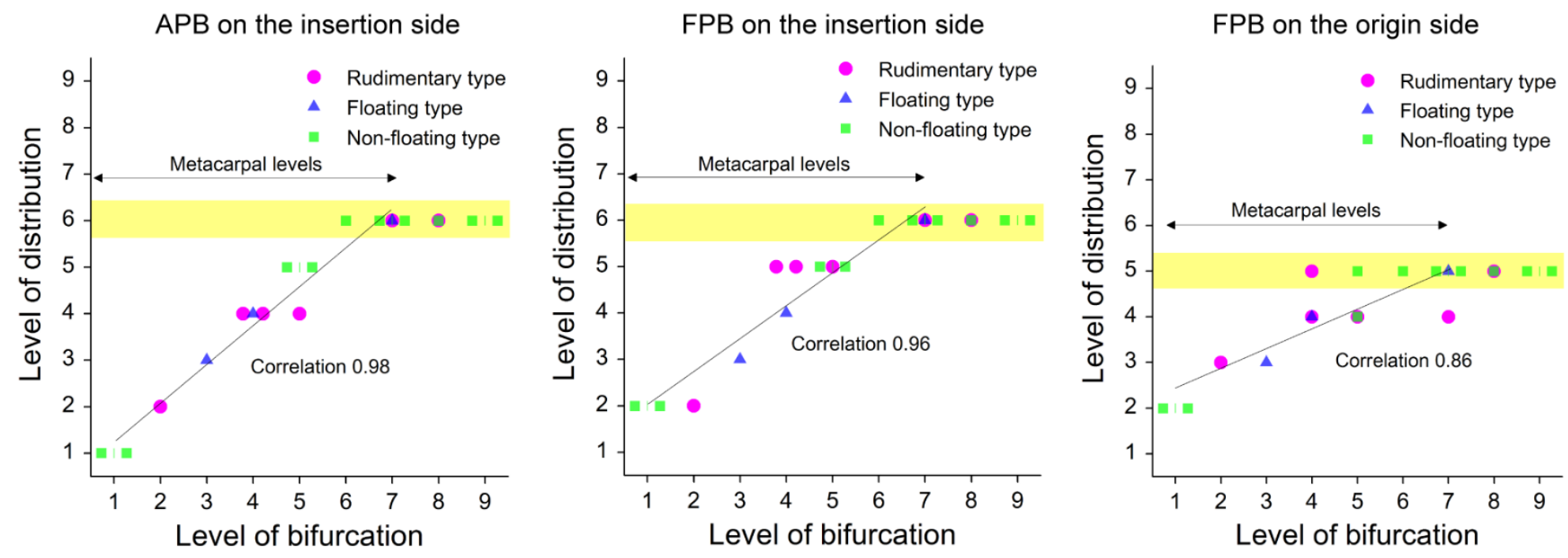
Fig. 3

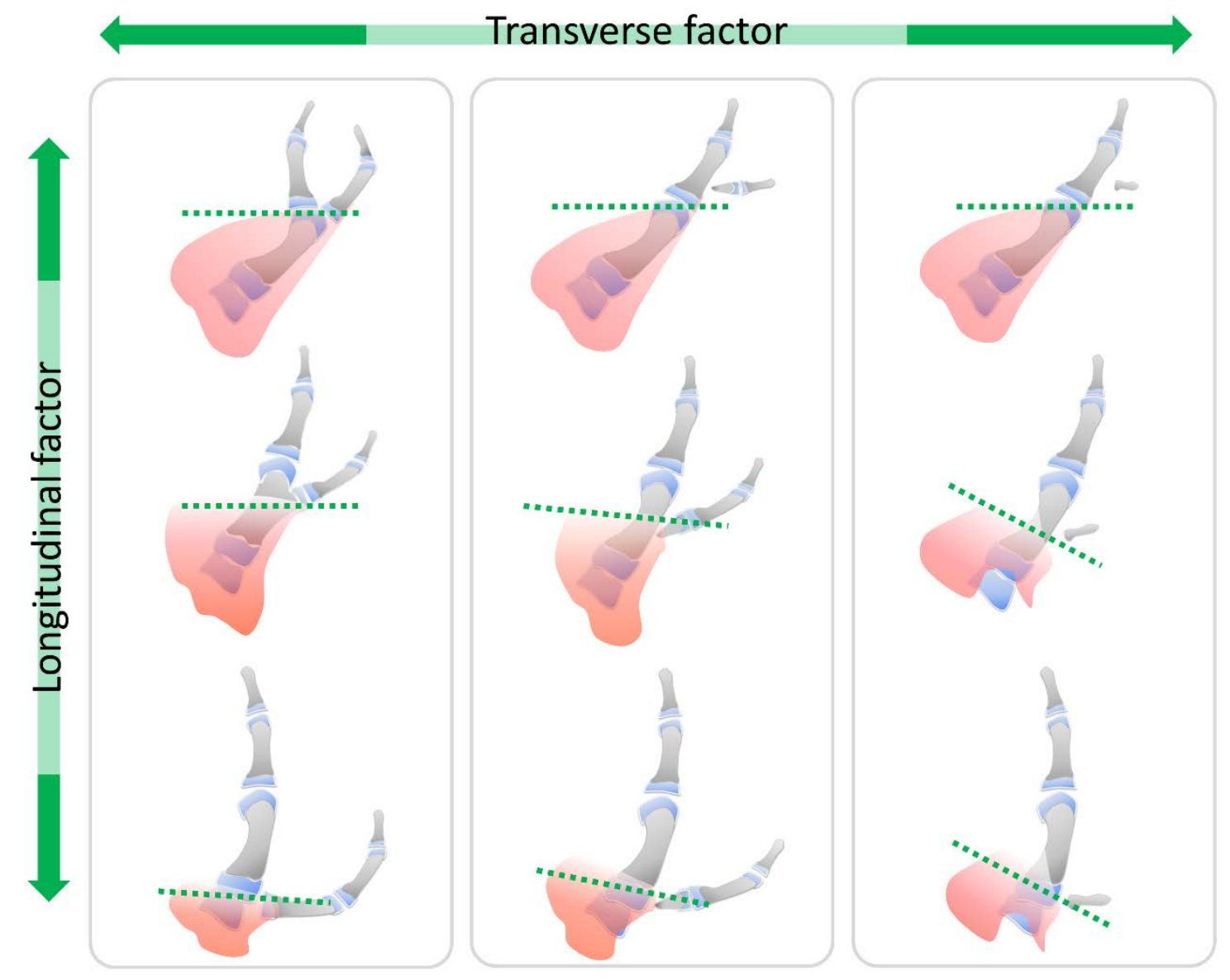


Supplementary figure 1

A

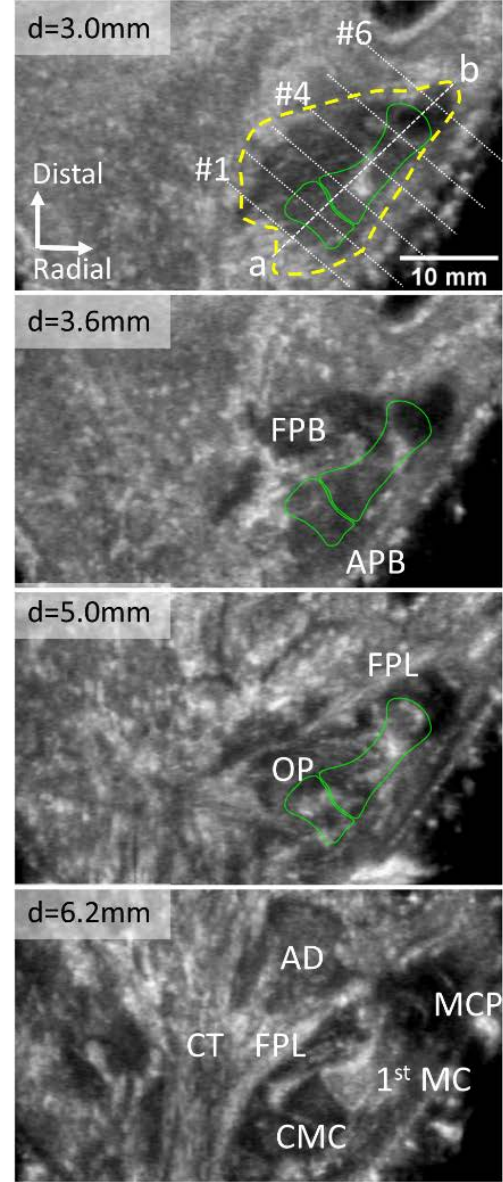

B

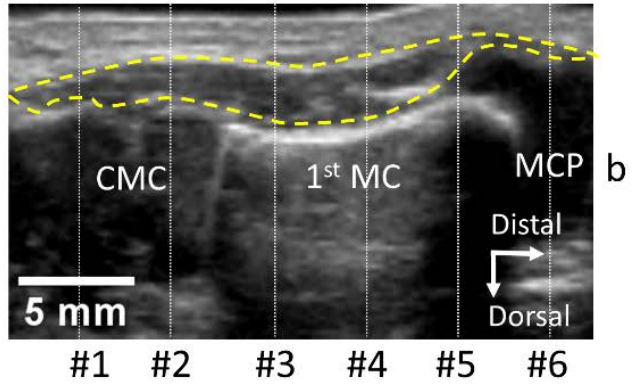

C
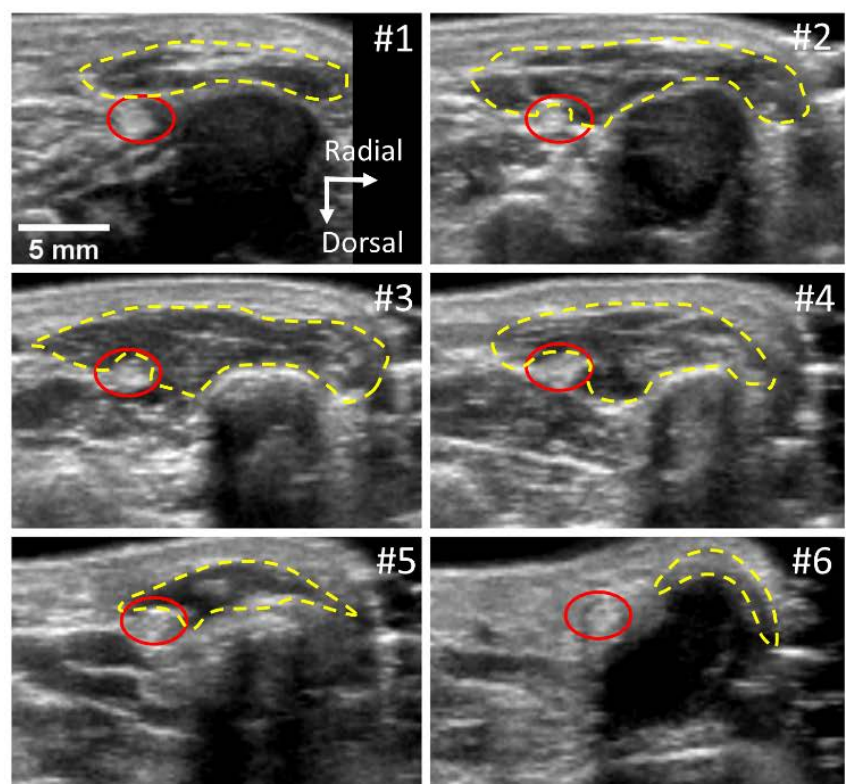
Supplementary figure 2

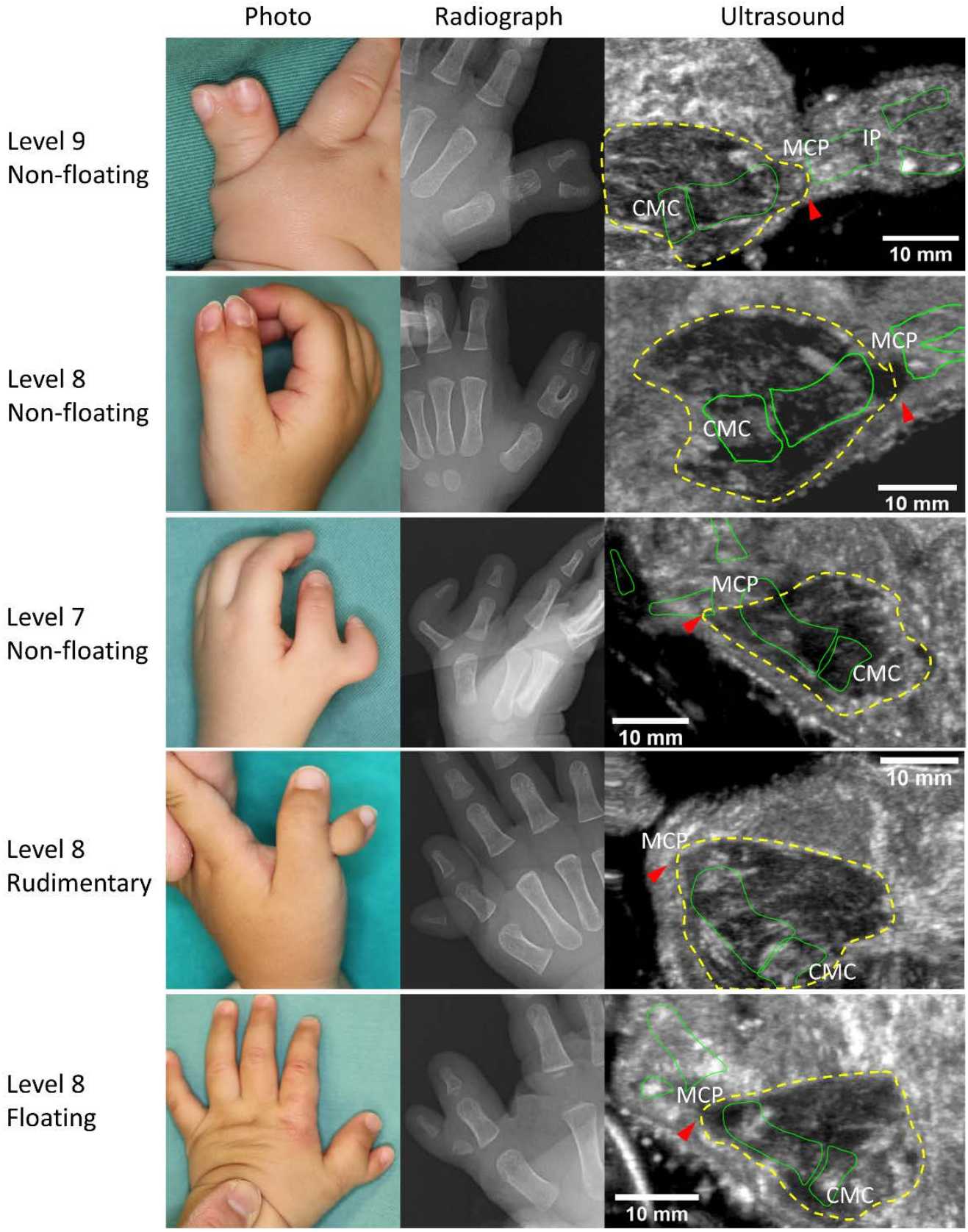


Supplementary figure 3

A

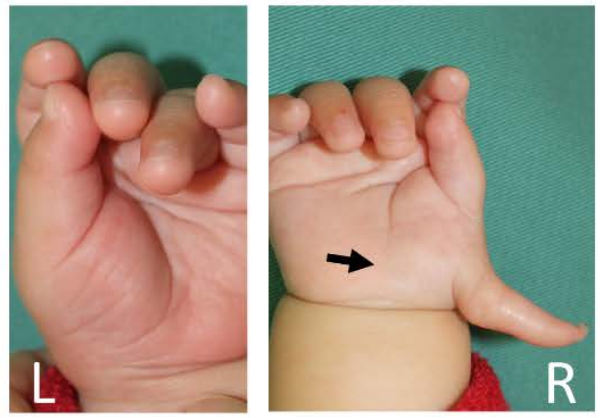

B

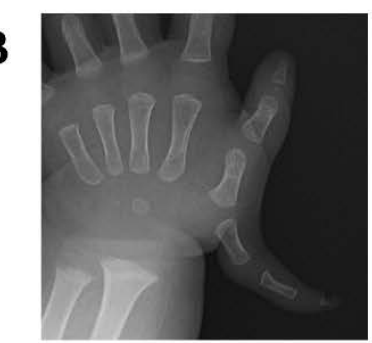

E

a

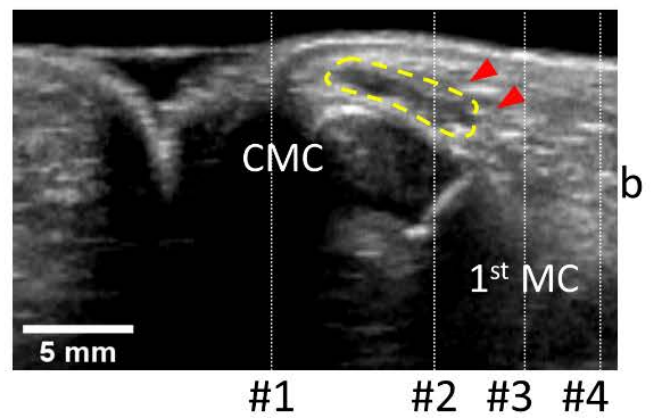

C

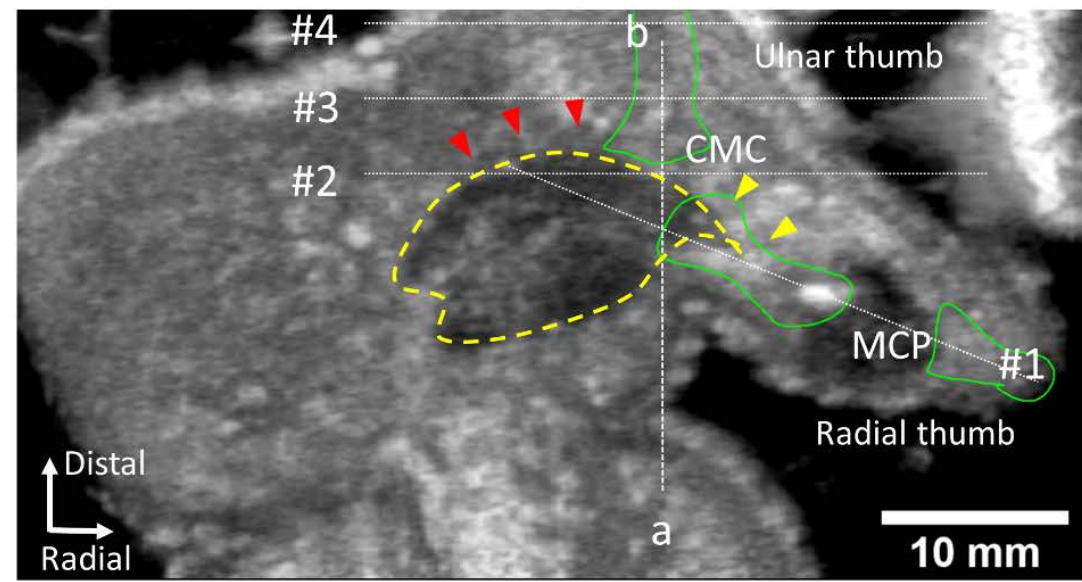

D

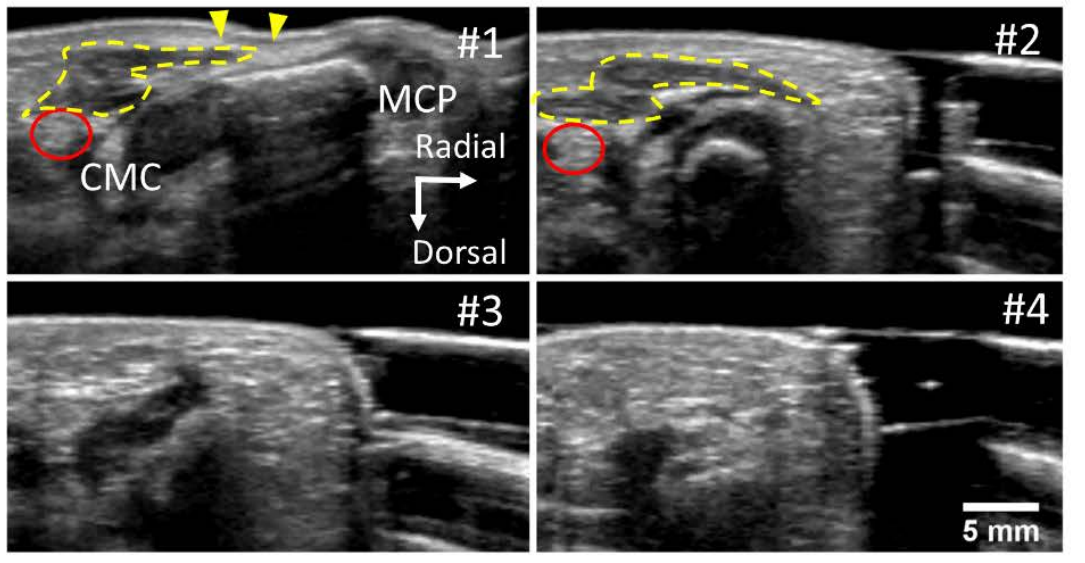


Supplementary figure 4

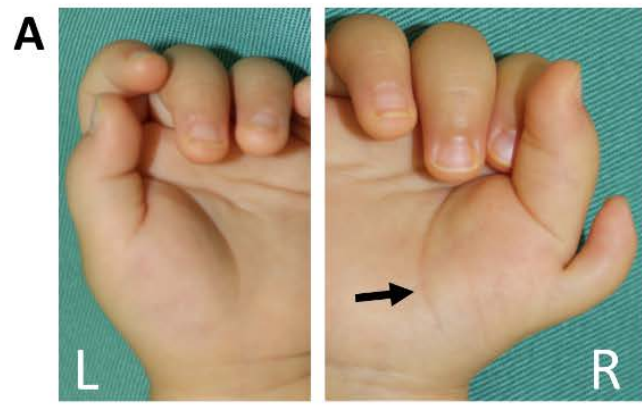

B

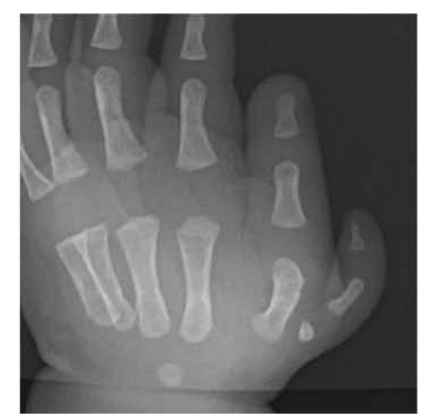

E

a

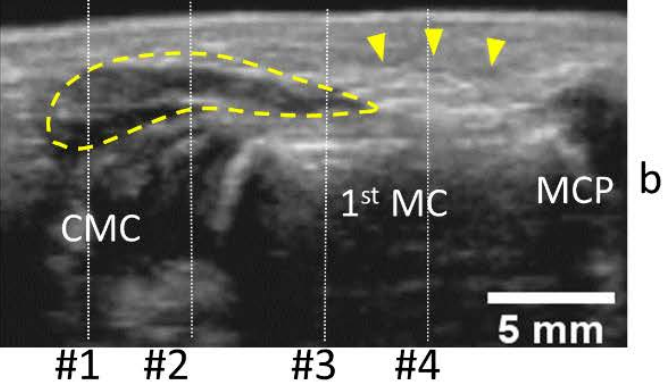

C

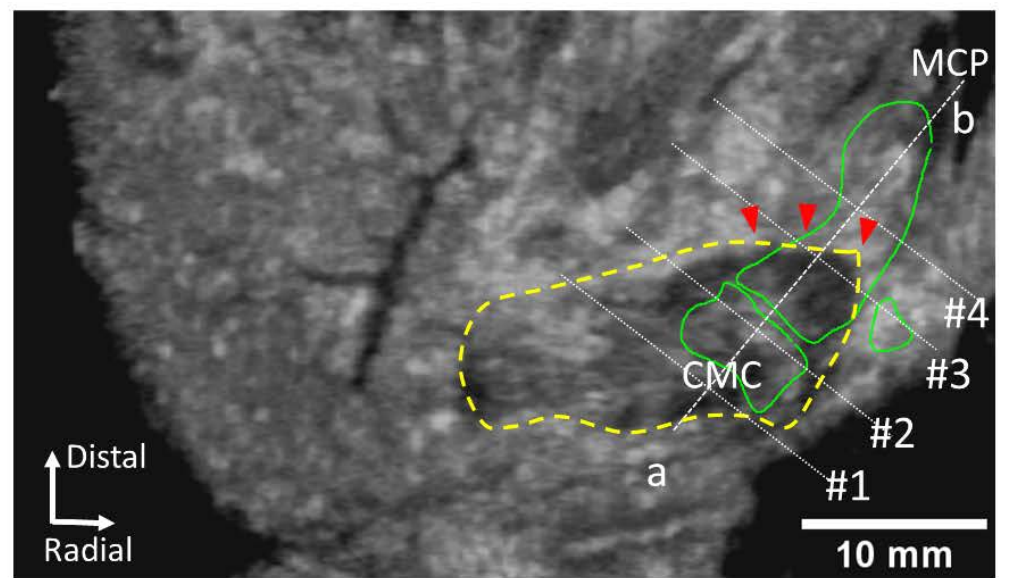

D
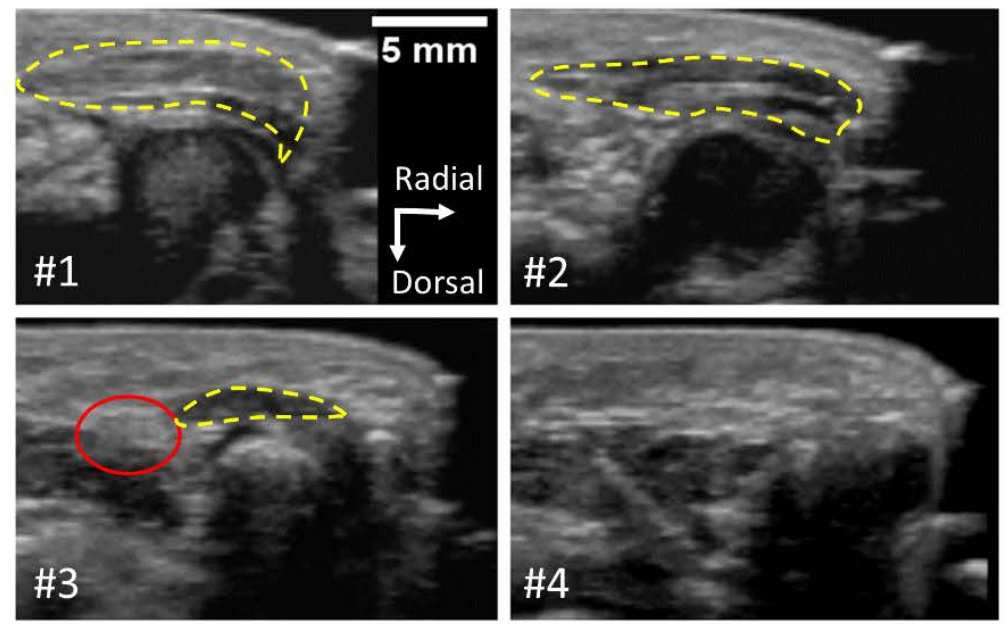

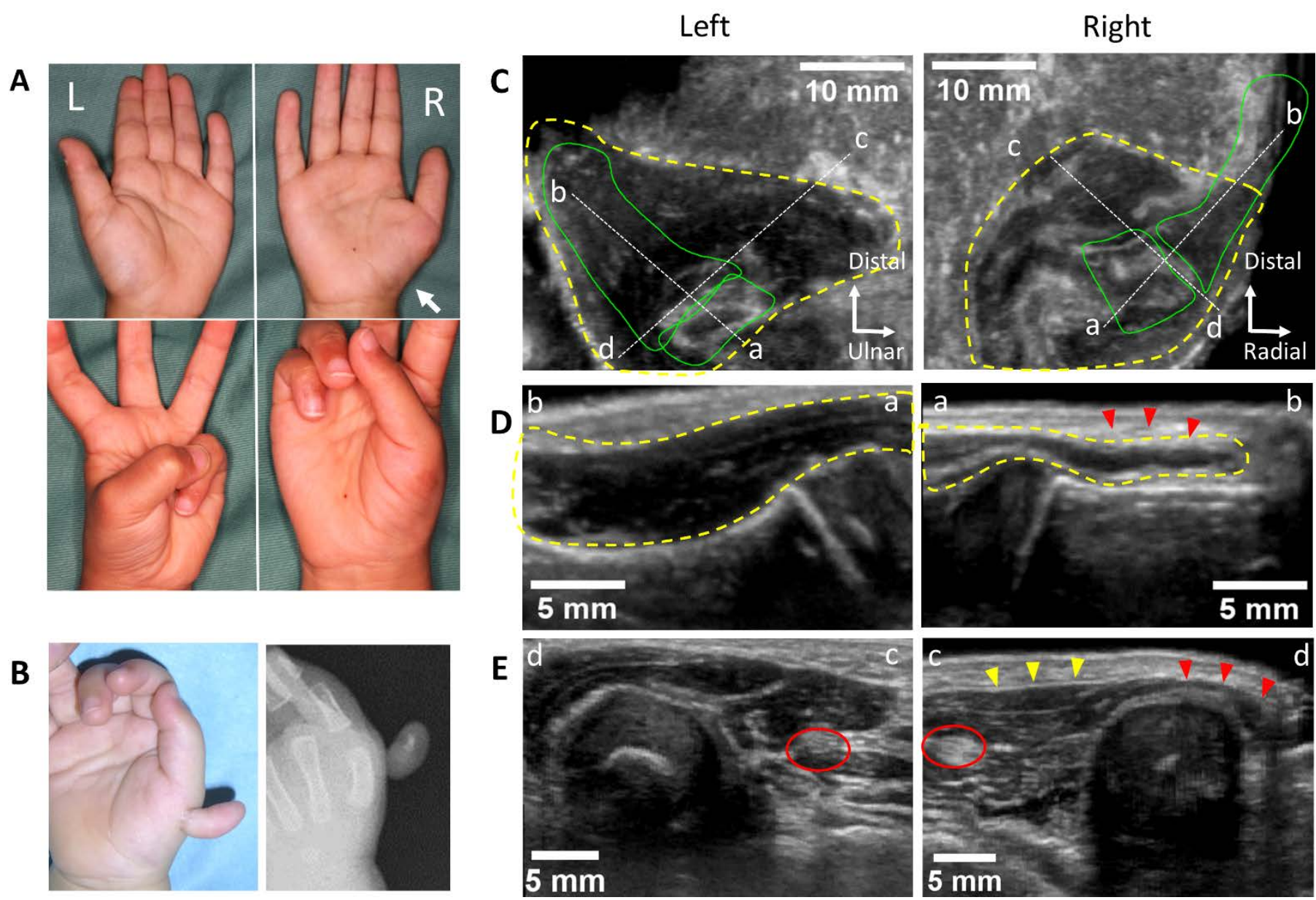

$5 \mathrm{~mm}$ 


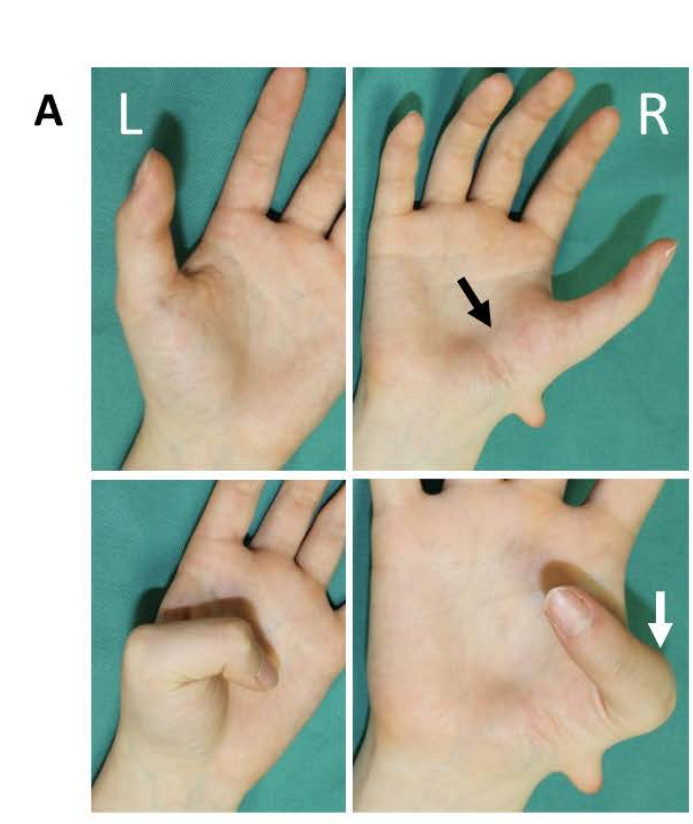

\section{B}
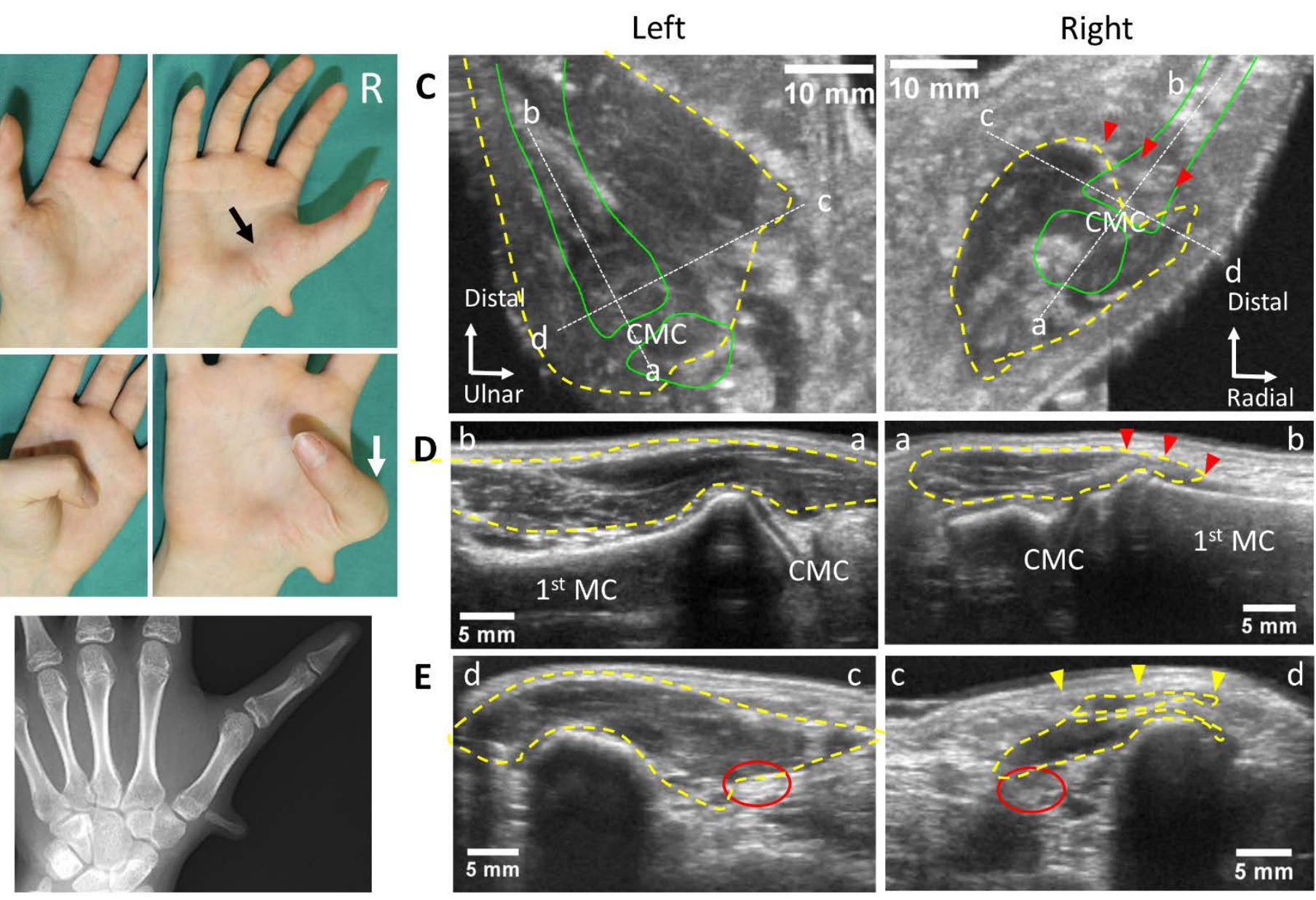